\title{
A Simple and Convenient Method for the Synthesis of 1-Methyl-7-arylfuro[3,2-g]pteridine-2,4(1H,3H)-diones and Their Substituted Derivatives
}

\author{
Maxim Stanislavovich Kazunin, ${ }^{1}$ Oleksii Yurievich Voskoboynik, ${ }^{1}$ \\ Svetlana Valentynivna Shishkina, ${ }^{2}$ Oleksii Mykolayovych Antypenko ${ }^{1}$ \\ and Sergey Ivanovich Kovalenko ${ }^{1,}$ \\ ${ }^{1}$ The Department of Organic and Bioorganic Chemistry, Zaporizhzhia State Medical University, \\ 26 Maiakovski Ave., Zaporizhzhia, 69035, Ukraine \\ 2 State Scientific Institution «Institute for Single Crystals» of National Academy of Sciences of Ukraine, \\ 60 Nauki Ave., Kharkov, 61000, Ukraine \\ *Corresponding author: E-mail: kovalenkosergiy@gmail.com
}

Received: 09-23-2019

\begin{abstract}
A simple and effective method for the synthesis of unknown 1-methyl-7-arylfuro[3,2-g]pteridine-2,4(1H,3H)-diones by dehydration of the corresponding 1-methyl-6-phenacyl-pteridine-2,4,7 $(1 H, 3 H, 8 H)$-triones is reported in the article. It was shown that their alkylation by butyl chloroacetate in basic medium proceeded by the $\mathrm{N}_{3}$-atom of the heterocycle. The structure and purity of the synthesized compounds were confirmed by $\mathrm{IR},{ }^{1} \mathrm{H},{ }^{13} \mathrm{C}$ NMR spectroscopy, gas chromatography-mass spectrometry, mass spectrometry, as well as X-ray diffraction analysis. The proposed mechanism of the dehydration reaction was discussed.
\end{abstract}

Keywords: 1-methyl-6-(2-oxo-2-arylethyl)pteridine-2,4,7(1H,3H,8H)-triones; dehydration; 1-methyl-7-arylfuro[3,2-g] pteridine-2,4(1H,3H)-diones; alkylation; X-ray diffraction analysis

\section{Introduction}

Condensed heterocyclic derivatives of pteridines belong to the important, but insufficiently studied group of organic substances. Although, the methods for the synthesis of annelated pteridines were systematized in few monographs ${ }^{1,2}$ and reviews, ${ }^{3-5}$ research devoted to the synthesis of condensed pteridines continues due to their high biological activity. Antimicrobial, ${ }^{6,7}$ antican$\mathrm{cer}^{8-10}$ anti-inflammatory and analgesic activities ${ }^{11,12}$ of condensed pteridines as well as their ability to inhibit PLK1 kinase ${ }^{13,14}$ have been described. Besides, these substances could be used as functional materials as shown in previous reports. ${ }^{15-17}$ The furo[3,2-g]pteridine system was not mentioned in the scientific literature, however methods for the isomeric furo[2,3-g]pteridines synthesis are known. ${ }^{3}$

Based on the above, the purpose of this work consists in developing a simple and convenient method for the synthesis of 1-methyl-7-arylfuro[3,2-g]pteridine-2,4(1H,3H) -diones by dehydration of 1-methyl-6-(2-oxo-2-arylethyl) pteridine-2,4,7(1H,3H,8H)-triones.

\section{Experimental Part}

\section{1. Chemistry}

Melting points were determined in open capillary tubes in a Mettler Toledo MP 50 apparatus and are uncorrected. The elemental analyses $(\mathrm{C}, \mathrm{H}, \mathrm{N})$ were performed using the ELEMENTAR vario EL cube analyzer (USA) and are within $\pm 0.3 \%$ of the theoretical values. IR spectra (4000-600 $\mathrm{cm}^{-1}$ ) were recorded on a Bruker ALPHA FTIR spectrometer (Bruker Bioscience, Germany) using a module for measuring attenuated total reflection (ATR). ${ }^{1} \mathrm{H}$ NMR spectra $(400 \mathrm{MHz})$ were recorded on a Varian-Mercury 400 (Varian Inc., Palo Alto, CA, USA) spectrometers with TMS as internal standard in DMSO- $d_{6}$ solution. ${ }^{13} \mathrm{C}$ NMR spectra of compounds $(\mathbf{3} \mathbf{b}-\mathbf{e}, \mathbf{3 g}-\mathbf{j}, 100$ $\mathrm{MHz}$ ) were recorded in TFA- $d_{1}$ solution. LC-MS were re- 
corded using chromatography/mass spectrometric system which consists of high performance liquid chromatography Agilent 1100 Series (Agilent, Palo Alto, CA, USA) equipped with diode-matrix and mass-selective detector Agilent LC/MSD SL (atmospheric pressure chemical ionization - APCI). Electron impact mass spectra (EI-MS) were recorded on a Varian $1200 \mathrm{~L}$ instrument at $70 \mathrm{eV}$ (Varian, USA).

Compounds 1a-k (1-methyl-6-(2-oxo-2-arylethyl) pteridine-2,4,7(1H,3H,8H)-triones) were obtained according to the previously described method. ${ }^{18}$ For the experiment commercially available reagents from Merck (Darmstadt, Germany), Sigma-Aldrich (Missouri, USA) and Enamine (Kyiv, Ukraine) were used.

General Method for the Synthesis of 1-Methyl-7-arylfuro $[3,2-g]$ pteridine-2,4(1H,3H)-diones $2 a-k$. A suspension of $10 \mathrm{mmol}$ of the corresponding 1-methyl-6-(2-oxo2-arylethyl)pteridine-2,4,7(1H,3H,8H)-trione $\mathbf{1 a}-\mathbf{k}$ in 20 $\mathrm{mL}$ of polyphosphoric acid was heated to $130^{\circ} \mathrm{C}$ and stirred for 1 hour. Afterwards, the reaction mixture was cooled, poured into $100 \mathrm{~mL}$ of water and stirred. The precipitate formed was filtered off, washed with water and dried.

1-Methyl-7-phenylfuro[3,2- $g$ ]pteridine-2,4(1H,3H)-dione (2a). Yield: $2.35 \mathrm{~g}$ (75\%), light yellow compound, $\mathrm{mp}$ $>300^{\circ} \mathrm{C}$; IR $\left(\mathrm{cm}^{-1}\right): 1698,1504,1340,1274,1178,1058$, 1010, 895, 831, 781, 761, 694, 670; ${ }^{1} \mathrm{H}$ NMR $\delta$ (ppm): 11.75 (s, $1 \mathrm{H}, 3-\mathrm{NH}), 8.01$ (d, $J=7.3 \mathrm{~Hz}, 2 \mathrm{H}, \mathrm{Ar} \mathrm{H}-2,6), 7.67$ (s, $1 \mathrm{H}, \mathrm{H}-6), 7.56-7.12$ (m, 3H, Ar H-3,4,5), 3.60 (s, 3H, 1-N$\mathrm{CH}_{3}$ ); EI-MS $m / z$ (I\% rel): 295 (13.6), $294\left(\mathrm{M}^{+}, 66.8\right), 251$ (12.6), 224 (18.2), 223 (100), 222 (11.5), 196 (11.9), 180 (5.6), 168 (5.2), 167 (5.9), 154 (16.9), 153 (8.3), 140 (24.8), 139 (9.8), 129 (8.4), 128 (11.0), 127 (24.5), 126 (9.6), 105 (27.6), 103 (12.7), 102 (21.8), 92 (10.1), 77 (25.3), 76 (6.9), 70 (12.6), 67 (16.8), 63 (5.0), 44 (76.2), 43 (15.9), 42 (6.9), 41 (10.6), 40 (7.0); LC-MS m/z=294 [M+H] $]^{+}$. Anal. Calcd. for $\mathrm{C}_{15} \mathrm{H}_{10} \mathrm{~N}_{4} \mathrm{O}_{3}: \mathrm{C}, 61.22 ; \mathrm{H}, 3.43 ; \mathrm{N}, 19.04$; found: $\mathrm{C}$, 61.27; H, 3.48; N, 19.09 .

1-Methyl-7-(para-tolyl)-furo[3,2-g]pteridine-2,4(1 H, $3 H$ )-dione (2b). Yield: $2.49 \mathrm{~g}(81 \%)$, light yellow compound, $\mathrm{mp}>300^{\circ} \mathrm{C}$; IR $\left(\mathrm{cm}^{-1}\right): 1681,1506,1342,1274$, $1179,1060,894,863,822,804,752,670,612 ;{ }^{1} \mathrm{H}$ NMR $\delta$ (ppm): $11.74(\mathrm{~s}, 1 \mathrm{H}, 3-\mathrm{NH}), 7.89(\mathrm{~d}, J=7.5 \mathrm{~Hz}, 2 \mathrm{H}, \mathrm{Ar}$ $\mathrm{H}-2,6), 7.57$ (s, $1 \mathrm{H}, \mathrm{H}-6), 7.34$ (d, $J=7.4 \mathrm{~Hz}, 2 \mathrm{H}, \mathrm{Ar} \mathrm{H}-3,5)$, $3.59\left(\mathrm{~s}, 3 \mathrm{H}, 1-\mathrm{N}-\mathrm{CH}_{3}\right), 2.44\left(\mathrm{~s}, 3 \mathrm{H}, \mathrm{ArCH}_{3}\right)$; LC-MS m/z= $308[\mathrm{M}+\mathrm{H}]^{+}$. Anal. Calcd. for $\mathrm{C}_{16} \mathrm{H}_{12} \mathrm{~N}_{4} \mathrm{O}_{3}: \mathrm{C}, 62.33 ; \mathrm{H}$, 3.92; N, 18.17; found: C, 62.39; H, 3.98; N, 18.22.

7-(4-Isopropylphenyl)-1-methylfuro[3,2-g]pteridine $\mathbf{- 2 , 4}(\mathbf{1 H}, \mathbf{3 H})$-dione (2c). Yield: $2.75 \mathrm{~g}(82 \%)$, light yellow compound, $\mathrm{mp}>300{ }^{\circ} \mathrm{C}$; IR $\left(\mathrm{cm}^{-1}\right): 1680,1503,1339$, 1270, 1059, 801, 746; ${ }^{1} \mathrm{H}$ NMR $\delta$ (ppm): 11.88 (s, $1 \mathrm{H}$, 3-NH), 7.91 (d, $J=7.5 \mathrm{~Hz}, 2 \mathrm{H}, \mathrm{Ar}-\mathrm{H}-2,6), 7.62(\mathrm{~s}, 1 \mathrm{H}$, H-6), 7.38 (d, J = 7.5 Hz, 2H, Ar H-3,5), 3.58 (s, 3H, 1-N-
$\left.\mathrm{CH}_{3}\right), 3.07-2.88\left(\mathrm{~m}, 1 \mathrm{H}, \mathrm{CH}\left(\mathrm{CH}_{3}\right)_{2}\right), 1.30(\mathrm{~d}, J=6.4 \mathrm{~Hz}$, $\left.6 \mathrm{H}, \mathrm{CH}\left(\mathrm{CH}_{3}\right)_{2}\right)$; LC-MS $m / z=336[\mathrm{M}+\mathrm{H}]^{+}$. Anal. Calcd. for $\mathrm{C}_{18} \mathrm{H}_{16} \mathrm{~N}_{4} \mathrm{O}_{3}$ : C, 64.28; $\mathrm{H}, 4.79 ; \mathrm{N}, 16.66$; found: $\mathrm{C}$, 64.33; H, 4.84; N, 16.71.

1-Methyl-7-(2-fluorophenyl)furo[3,2-g]pteridine-2,4 (1H,3H)-dione (2d). Yield: $2.41 \mathrm{~g}(77 \%)$, light yellow compound, $\mathrm{mp}>300{ }^{\circ} \mathrm{C}$; IR $\left(\mathrm{cm}^{-1}\right): 1696,1488,1338$, $1263,1175,1056,1008,895,808,777,762,745,652 ;{ }^{1} \mathrm{H}$ NMR $\delta(\mathrm{ppm}): 11.96(\mathrm{~s}, 1 \mathrm{H}, 3-\mathrm{NH}), 8.24-8.02(\mathrm{~m}, 1 \mathrm{H}, \mathrm{Ar}$ H-6), 7.70-7.50 (m, 2H, H-6, Ar H-4), 7.47-7.28 (m, 2H, Ar H-3,5), 3.60 (s, 3H, 1-N-CH ${ }_{3}$ ); LC-MS $m / z=312$ $[\mathrm{M}+\mathrm{H}]^{+}$. Anal. Calcd. for $\mathrm{C}_{15} \mathrm{H}_{9} \mathrm{FN}_{4} \mathrm{O}_{3}: \mathrm{C}, 57.70 ; \mathrm{H}, 2.91$; N, 17.94; found: C, 57.76; H, 2.30; N, 17.99 .

1-Methyl-7-(4-fluorophenyl)furo[3,2-g]pteridine-2,4 $(1 \mathrm{H}, 3 \mathrm{H})$-dione (2e). Yield: $2.55 \mathrm{~g}(82 \%)$, light yellow compound, $\mathrm{mp}>300^{\circ} \mathrm{C}$; IR $\left(\mathrm{cm}^{-1}\right): 3048,1713,1683,1603$, $1505,1435,1345,1274,1236,1162,1059,893,843,805$, 747, 611; ${ }^{1} \mathrm{H}$ NMR $\delta$ (ppm): 11.91 (s, 1H, 3-NH), 8.108.02 (m, 2H, Ar H-2,6), 7.73 (s, 1H, H-6), 7.37-7.21 (m, $2 \mathrm{H}, \mathrm{Ar} \mathrm{H}-3,5), 3.57$ (s, 3H, 1-N-CH ${ }_{3}$; LC-MS $m / z=312$ $[\mathrm{M}+\mathrm{H}]^{+}$. Anal. Calcd. for $\mathrm{C}_{15} \mathrm{H}_{9} \mathrm{FN}_{4} \mathrm{O}_{3}$ : C 57.70; $\mathrm{H}, 2.91$; N, 17.94; found: C, 57.76; H, 2.97; N, 17.98 .

7-(2,4-Difluorophenyl)-1-methylfuro[3,2-g]pteridine-2,4 $(\mathbf{1 H}, \mathbf{3 H})$-dione (2f). Yield: $2.57 \mathrm{~g}(78 \%)$, light yellow compound, $\mathrm{mp}>300{ }^{\circ} \mathrm{C} ;{ }^{1} \mathrm{H}$ NMR $\delta$ (ppm): 11.83 (s, 1H, 3-NH), 8.18-7.96 (m, 2H, Ar H-6), 7.49 (s, 1H, $\mathrm{H}-6), 7.33-7.08(\mathrm{~m}, 2 \mathrm{H}, \mathrm{Ar} 3,5), 3.58\left(\mathrm{~s}, 3 \mathrm{H}, 1-\mathrm{N}-\mathrm{CH}_{3}\right)$; LC-MS $m / z=330[\mathrm{M}+\mathrm{H}]^{+}$. Anal. Calcd. for $\mathrm{C}_{15} \mathrm{H}_{8} \mathrm{~F}_{2} \mathrm{~N}_{4} \mathrm{O}_{3}$ : C, 54.55; H, 2.44; N, 16.97; found: C, $54.61 ; \mathrm{H}, 2.50 ; \mathrm{N}$, 17.02 .

1-Methyl-7-(4-chlorophenyl)-furo[3,2-g]pteridine-2,4 $(\mathbf{1 H}, 3 \mathrm{H})$-dione $(2 \mathrm{~g})$. Yield: $2.59 \mathrm{~g}(79 \%)$, light yellow compound, $\mathrm{mp}>300{ }^{\circ} \mathrm{C}$; IR $\left(\mathrm{cm}^{-1}\right): 3047,1716,1689,1504$, 1433, 1342, 1288, 1177, 1090, 1059, 1003, 893, 841, 826, 805,$751 ;{ }^{1} \mathrm{H}$ NMR $\delta$ (ppm): 11.86 (s, $\left.1 \mathrm{H}, 3-\mathrm{NH}\right), 8.03$ (d, $J$ $=8.9 \mathrm{~Hz}, 2 \mathrm{H}, \mathrm{Ar} \mathrm{H}-2,6), 7.78(\mathrm{~s}, 1 \mathrm{H}, \mathrm{H}-6), 7.54(\mathrm{~d}, J=9.3$ $\mathrm{Hz}, 2 \mathrm{H}, \mathrm{Ar} \mathrm{H}-3,5), 3.58$ (s, 3H, 1-N-CH $)$; LC-MS $m / z=$ $328[\mathrm{M}+\mathrm{H}]^{+}$. Anal. Calcd. for $\mathrm{C}_{15} \mathrm{H}_{9} \mathrm{ClN}_{4} \mathrm{O}_{3}: \mathrm{C}, 54.81 ; \mathrm{H}$, 2.76; N, 17.04; found: C, 54.88; H, 2.81; N, 17.09 .

7-(4-Bromophenyl)-1-methylfuro[3,2-g]pteridine-2,4 (1H,3H)-dione (2h). Yield: $3.09 \mathrm{~g}(83 \%)$, light yellow compound, $\mathrm{mp}>300^{\circ} \mathrm{C}$; IR $\left(\mathrm{cm}^{-1}\right): 3054,1691,1503$, 1340, 1286, 1059, 1001, 893, 837, 822, 803, 749; ${ }^{1} \mathrm{H}$ NMR $\delta$ (ppm): $11.44(\mathrm{~s}, 1 \mathrm{H}, 3-\mathrm{NH}), 7.98(\mathrm{~d}, J=7.3 \mathrm{~Hz}, 2 \mathrm{H}, \mathrm{Ar}$ $\mathrm{H}-2,6), 7.84$ (s, $1 \mathrm{H}, \mathrm{H}-6), 7.71$ (d, $J=7.4 \mathrm{~Hz}, 2 \mathrm{H}, \mathrm{Ar} \mathrm{H}-3,5)$, 3.59 (s, 3H, $\left.1-\mathrm{N}-\mathrm{CH}_{3}\right)$; LC-MS $\mathrm{m} / z=373[\mathrm{M}+\mathrm{H}]^{+}$. Anal. Calcd. for $\mathrm{C}_{15} \mathrm{H}_{9} \mathrm{BrN}_{4} \mathrm{O}_{3}: \mathrm{C}, 48.28 ; \mathrm{H}, 2.43 ; \mathrm{N}, 15.01$; found: $\mathrm{C}, 48.32 ; \mathrm{H}, 2.49 ; \mathrm{N}, 15.08$.

1-Methyl-7-(3-nitrophenyl)furo[3,2-g]pteridine-2,4 $(\mathbf{H}, 3 \mathrm{H})$-dione (2i). Yield: $2.74 \mathrm{~g}(81 \%)$, light brown com- 
pound, $\mathrm{mp}>300{ }^{\circ} \mathrm{C}$; IR $\left(\mathrm{cm}^{-1}\right): 1681,1527,1503,1342$, $1291,1270,1187,1059,914,850,807,740,725,681 ;{ }^{1} \mathrm{H}$ NMR $\delta$ (ppm): 11.98 (s, 1H, 3-NH), 8.86 (s, 1H, Ar H-2), $8.48(\mathrm{~d}, J=8.6 \mathrm{~Hz}, 1 \mathrm{H}, \mathrm{Ar} \mathrm{H}-6), 8.33(\mathrm{~d}, J=7.4 \mathrm{~Hz}, 1 \mathrm{H}, \mathrm{Ar}$ H-4), 8.14 (s, 1H, H-6), 7.86 (t, $J=8.8 \mathrm{~Hz}, 1 \mathrm{H}, \mathrm{Ar} \mathrm{H}-5)$, $3.60\left(\mathrm{~s}, 3 \mathrm{H}, 1-\mathrm{N}-\mathrm{CH}_{3}\right)$; LC-MS $m / z=339[\mathrm{M}+\mathrm{H}]^{+}$. Anal. Calcd. for $\mathrm{C}_{15} \mathrm{H}_{9} \mathrm{~N}_{5} \mathrm{O}_{5}$ : C, 53.10; H, 2.67; N, 20.64; found C, 53.17; H, 2.73; N, 20.69 .

1-Methyl-7-(naphthalen-2-yl)-furo[3,2-g]pteridine-2,4 $(\mathbf{1 H}, \mathbf{3 H})$-dione (2j). Yield: $2.85 \mathrm{~g}(83 \%)$, light orange substance, $\mathrm{mp}>300{ }^{\circ} \mathrm{C}$; IR $\left(\mathrm{cm}^{-1}\right): 3047,1728,1674,1507$, 1468, 1346, 1283, 1266, 1215, 1060, 947, 833, 748, 711, 662; ${ }^{1} \mathrm{H}$ NMR $\delta$ (ppm): 12.03 (s, $\left.1 \mathrm{H}, 3-\mathrm{NH}\right), 8.80$ (s, $1 \mathrm{H}$, naphthalene $\mathrm{H}-1), 8.11-7.14(\mathrm{~m}, 7 \mathrm{H}, \mathrm{H}-6$, naphthalene $\mathrm{H}-3,4,5,6,7,8)$, 3.61 (s, $3 \mathrm{H}, 1-\mathrm{N}-\mathrm{CH}_{3}$ ); LC-MS $m / z=344$ $[\mathrm{M}+\mathrm{H}]^{+}$. Anal. Calcd. for $\mathrm{C}_{19} \mathrm{H}_{12} \mathrm{~N}_{4} \mathrm{O}_{3}$ : C, 66.28; H, 3.51; $\mathrm{N}, 16.2$; found: $\mathrm{C}, 66.34 ; \mathrm{H}, 3.58 ; \mathrm{N}, 16.32$.

7-(4-Methoxyphenyl)-1-methylfuro[3,2-g]pteridine-2,4 $(\mathbf{1 H}, 3 \mathrm{H})$-dione (2k). Yield: $2.65 \mathrm{~g}(82 \%)$, light yellow compound, $\mathrm{mp}>300{ }^{\circ} \mathrm{C}$; IR $\left(\mathrm{cm}^{-1}\right): 1714,1681,1600$, 1504, 1340, 1286, 1259, 1175, 1057, 1014, 893, 843, 798, 748,$614 ;{ }^{1} \mathrm{H}$ NMR $\delta$ (ppm): $11.86(\mathrm{~s}, 1 \mathrm{H}, 3-\mathrm{NH}), 7.95$ (d, $J$ $=7.3 \mathrm{~Hz}, 2 \mathrm{H}, \mathrm{Ar} \mathrm{H}-2,6), 7.53(\mathrm{~s}, 1 \mathrm{H}, \mathrm{H}-6), 7.06$ (d, $J=8.8$ $\mathrm{Hz}, 2 \mathrm{H}, \mathrm{Ar} \mathrm{H}-3,5), 3.88\left(\mathrm{~s}, 3 \mathrm{H}, \mathrm{OCH}_{3}\right), 3.57$ (s, 3H, $\left.1-\mathrm{N}-\mathrm{CH}_{3}\right)$; LC-MS $m / z=324[\mathrm{M}+\mathrm{H}]^{+}$. Anal. Calcd. for $\mathrm{C}_{16} \mathrm{H}_{12} \mathrm{~N}_{4} \mathrm{O}_{4}$ : C, 59.26; $\mathrm{H}, 3.73 ; \mathrm{N}, 17.28$; found: $\mathrm{C}, 59.31$; $\mathrm{H}, 3.79 ; \mathrm{N}, 17.32$.

General Method for the Synthesis of Butyl 2-(7-Aryl -2,4-dioxo-1-methyl-1,4-dihydrofuro[3,2-g]pteridine$3(2 \mathrm{H})$-yl)acetates $3 \mathbf{a}-\mathbf{j}$. To a suspension of $10 \mathrm{mmol}$ of the corresponding 1-methyl-7-arylfuro[3,2-g]pteridine-2,4 $(1 \mathrm{H}, 3 \mathrm{H})$-dione $\mathbf{2 a}-\mathbf{k}$ and $10 \mathrm{mmol}$ of $\mathrm{K}_{2} \mathrm{CO}_{3}$ in $30 \mathrm{~mL}$ of dimethylformamide, $10 \mathrm{mmol}$ of butyl chloroacetate was added and refluxed for 2 hours. The reaction mixture was cooled, poured into $100 \mathrm{~mL}$ of water and stirred. The precipitate formed was filtered off, washed with water, dried and crystallized from dimethylformamide.

Butyl 2-(2,4-Dioxo-1-methyl-7-phenyl-1,4-dihydrofuro[3,2-g]pteridine-3(2H)-yl)acetate (3a). Yield: $3.34 \mathrm{~g}$ (81\%), light yellow compound, mp: $297-299^{\circ} \mathrm{C}$; IR $\left(\mathrm{cm}^{-1}\right)$ : $1750,1715,1675,1562,1508,1360,1279,1200,1008,931$, $893,803,770,755,688 ;{ }^{1} \mathrm{H}$ NMR $\delta(\mathrm{ppm}): 8.03(\mathrm{~d}, J=7.3$ $\mathrm{Hz}, 2 \mathrm{H}, \mathrm{Ar} \mathrm{H}-2,6), 7.78$ (s, 1H, H-6), 7.62-7.46 (m, 3H, Ar $\mathrm{H}-3,4,5), 4.73\left(\mathrm{~s}, 2 \mathrm{H}, \mathrm{NCH}_{2}\right), 4.15\left(\mathrm{t}, J=6.5 \mathrm{~Hz}, 2 \mathrm{H}, \mathrm{OCH}_{2}\right.$ $\left.\mathrm{CH}_{2} \mathrm{CH}_{2} \mathrm{CH}_{3}\right), 3.69$ (s, $\left.3 \mathrm{H}, 1-\mathrm{N}-\mathrm{CH}_{3}\right), 1.78-1.52(\mathrm{~m}, 2 \mathrm{H}$, $\left.\mathrm{OCH}_{2} \mathrm{CH}_{2} \mathrm{CH}_{2} \mathrm{CH}_{3}\right), 1.52-1.20\left(\mathrm{~m}, 2 \mathrm{H}, \mathrm{OCH}_{2} \mathrm{CH}_{2} \mathrm{CH}_{2}\right.$ $\mathrm{CH}_{3}$ ), 0.95 (t, $J=7.3 \mathrm{~Hz}, 3 \mathrm{H}, \mathrm{OCH}_{2} \mathrm{CH}_{2} \mathrm{CH}_{2} \mathrm{CH}_{3}$ ); EI-MS $\mathrm{m} / z$ (I\% rel): 409 (11.6), 408 (46.6), 352 (10.6), 309 (8.0), 308 (31.9), 307 (30.2), 279 (8.7), 251 (9.5), 249 (5.3), 224 (60), 223 (23.9), 167 (6.4), 140 (10.2), 105 (8.8), 86 (34.3), 84 (49.5), 83 (8.9), 57 (17.3), 56 (18.4), 51 (29.5), 50 (6.8), 49 (100), 48 (11.8), 47 (15.6), 44 (8.9), 43 (6.8), 42 (5.9), 41
(36.4); LC-MS $m / z=408[\mathrm{M}+\mathrm{H}]^{+}$. Anal. Calcd. for $\mathrm{C}_{21} \mathrm{H}_{20}$ $\mathrm{N}_{4} \mathrm{O}_{5}: \mathrm{C}, 61.76 ; \mathrm{H}, 4.94 ; \mathrm{N}, 13.72$; found $\mathrm{C}, 61.81 ; \mathrm{H}, 5.01$; $\mathrm{N}, 13.77$.

Butyl 2-(2,4-Dioxo-1-methyl-7-(p-tolyl)-1,4-dihydrofuro[3,2-g]pteridine-3(2H)-yl)acetate $(3 \mathrm{~b})$. Yield: $3.21 \mathrm{~g}$ (76\%), light yellow compound, $\mathrm{mp}: 295-297^{\circ} \mathrm{C}$; IR $\left(\mathrm{cm}^{-1}\right)$ : 1754, 1714, 1674, 1505, 1453, 1360, 1279, 1195, 1000, 932, 892, 818, 801, 756; ${ }^{1} \mathrm{H}$ NMR $\delta$ (ppm): 7.93 (d, $J=7.5 \mathrm{~Hz}$, 2H, Ar H-2,6), 7.69 (s, $1 \mathrm{H}, \mathrm{H}-6), 7.36$ (d, J = 6.9 Hz, 2H, Ar $\mathrm{H}-3,5), 4.74\left(\mathrm{~s}, 2 \mathrm{H}, \mathrm{NCH}_{2}\right), 4.17\left(\mathrm{t}, J=6.4 \mathrm{~Hz}, 2 \mathrm{H}, \mathrm{OCH}_{2}\right.$ $\mathrm{CH}_{2} \mathrm{CH}_{2} \mathrm{CH}_{3}$ ), 3.70 (s, $\left.3 \mathrm{H}, 1-\mathrm{N}-\mathrm{CH}_{3}\right), 2.46$ (s, $\left.3 \mathrm{H}, \mathrm{ArCH}_{3}\right)$, 1.86-1.55 (m, 2H, OCH $\left.{ }_{2} \mathrm{CH}_{2} \mathrm{CH}_{2} \mathrm{CH}_{3}\right), 1.46-1.36(\mathrm{~m}, 2 \mathrm{H}$, $\left.\mathrm{OCH}_{2} \mathrm{CH}_{2} \mathrm{CH}_{2} \mathrm{CH}_{3}\right), 0.97\left(\mathrm{t}, J=6.8 \mathrm{~Hz}, 3 \mathrm{H}, \mathrm{OCH}_{2} \mathrm{CH}_{2}\right.$ $\left.\mathrm{CH}_{2} \mathrm{CH}_{3}\right) ;{ }^{13} \mathrm{C}$ NMR $\delta$ (ppm): 172.7 (COO), $170.1(\mathrm{C}-8 \mathrm{a})$, 157.5 (C-4), 150.3 (C-7), 148.3 (C-2), 145.7 (C-9a), 135.5 (C-5a), 130.4 (Ar C-2,6), 127.8 (Ar C-3,5), 122.5 (Ar C-1), 117.6 (C-4a), 113.8 (Ar C-4), 95.1 (C-6), $68.2\left(\mathrm{OCH}_{2} \mathrm{CH}_{2}\right.$ $\left.\mathrm{CH}_{2} \mathrm{CH}_{3}\right), 43.5\left(\mathrm{NCH}_{2} \mathrm{CO}\right), 30.0\left(\mathrm{~N}-\mathrm{CH}_{3}\right), 29.7\left(\mathrm{OCH}_{2}\right.$ $\left.\mathrm{CH}_{2} \mathrm{CH}_{2} \mathrm{CH}_{3}\right), 20.2\left(\mathrm{Ar}-\mathrm{CH}_{3}\right), 18.2\left(\mathrm{OCH}_{2} \mathrm{CH}_{2} \mathrm{CH}_{2} \mathrm{CH}_{3}\right)$, $11.7\left(\mathrm{OCH}_{2} \mathrm{CH}_{2} \mathrm{CH}_{2} \mathrm{CH}_{3}\right)$; LC-MS $m / z=422[\mathrm{M}+\mathrm{H}]^{+}$. Anal. Calcd. for $\mathrm{C}_{22} \mathrm{H}_{22} \mathrm{~N}_{4} \mathrm{O}_{5}$ : C, 62.55; H, 5.25; N, 13.26; found: $\mathrm{C}, 62.59 ; \mathrm{H}, 5.29 ; \mathrm{N}, 13.31$.

Butyl 2-(2,4-Dioxo-7-(4-isopropylphenyl)-1-methyl-1,4 -dihydrofuro[3,2-g]pteridine-3(2H)-yl)acetate (3c). Yield: $3.46 \mathrm{~g}$ (77\%), light yellow compound, mp: 288-291 ${ }^{\circ} \mathrm{C}$; IR $\left(\mathrm{cm}^{-1}\right)$ : 2957, 1761, 1724, 1667, 1512, 1455, 1362, 1282, 1157, 1006, 894, 843, 805, 755; ${ }^{1} \mathrm{H}$ NMR $\delta(\mathrm{ppm})$ : 7.95 (d, $J=8.1 \mathrm{~Hz}, 2 \mathrm{H}, \mathrm{Ar} \mathrm{H}-2,6), 7.69$ (s, $1 \mathrm{H}, \mathrm{H}-6), 7.40$ $(\mathrm{d}, J=8.1 \mathrm{~Hz}, 2 \mathrm{H}, \mathrm{Ar} \mathrm{H}-3,5), 4.74\left(\mathrm{~s}, 2 \mathrm{H}, \mathrm{NCH}_{2}\right), 4.16$ (t, $J$ $\left.=6.6 \mathrm{~Hz}, 2 \mathrm{H}, \mathrm{OCH}_{2} \mathrm{CH}_{2} \mathrm{CH}_{2} \mathrm{CH}_{3}\right), 3.70\left(\mathrm{~s}, 3 \mathrm{H}, 1-\mathrm{N}-\mathrm{CH}_{3}\right)$, 3.16-2.62 (m, $\left.1 \mathrm{H}, \mathrm{CH}\left(\mathrm{CH}_{3}\right)_{2}\right), 1.66\left(\mathrm{~m}, 2 \mathrm{H}, \mathrm{OCH}_{2} \mathrm{CH}_{2}\right.$ $\left.\mathrm{CH}_{2} \mathrm{CH}_{3}\right), 1.41\left(\mathrm{~m}, 2 \mathrm{H}, \mathrm{OCH}_{2} \mathrm{CH}_{2} \mathrm{CH}_{2} \mathrm{CH}_{3}\right), 1.31(\mathrm{~d}, \mathrm{~J}=$ $\left.6.8 \mathrm{~Hz}, 6 \mathrm{H}, \mathrm{CH}\left(\mathrm{CH}_{3}\right)_{2}\right), 0.97\left(\mathrm{t}, J=7.3 \mathrm{~Hz}, 3 \mathrm{H}, \mathrm{OCH}_{2} \mathrm{CH}_{2}\right.$ $\mathrm{CH}_{2} \mathrm{CH}_{3}$ ); ${ }^{13} \mathrm{C}$ NMR $\delta$ (ppm): 168.1 (COO, C-8a), 161.4 (C-4), 159.1 (C-7), 155.7 (Ar C-4), 152.2 (C-2), 150.1 (C9a), 145.6 (C-5a), 139.0 (C-4a), 127.7 (Ar C-2,6), 125.9 (Ar C-3,5), 125.0 (Ar C-1), 116.0 (C-4a), 101.4 (C-6), 65.3 $\left(\mathrm{OCH}_{2} \mathrm{CH}_{2} \mathrm{CH}_{2} \mathrm{CH}_{3}\right), 43.2\left(\mathrm{NCH}_{2} \mathrm{CO}\right), 33.9\left(\underline{\mathrm{CH}}\left(\mathrm{CH}_{3}\right)_{2}\right)$, $30.5\left(\mathrm{OCH}_{2} \mathrm{CH}_{2} \mathrm{CH}_{2} \mathrm{CH}_{3}\right), \quad 30.0\left(\mathrm{~N}-\mathrm{CH}_{3}\right), 23.9(\mathrm{CH}$ $\left.\left(\mathrm{CH}_{3}\right)_{2}\right), 18.9\left(\mathrm{OCH}_{2} \mathrm{CH}_{2} \mathrm{CH}_{2} \mathrm{CH}_{3}\right), 13.9\left(\mathrm{OCH}_{2} \mathrm{CH}_{2} \mathrm{CH}_{2}\right.$ $\left.\mathrm{CH}_{3}\right) ; \mathrm{LC}-\mathrm{MS} m / z=450[\mathrm{M}+\mathrm{H}]^{+}$. Anal. Calcd. for $\mathrm{C}_{24} \mathrm{H}_{26}$ $\mathrm{N}_{4} \mathrm{O}_{5}$ : C, 63.99; $\mathrm{H}, 5.82 ; \mathrm{N}, 12.44$; found: $\mathrm{C}, 64.04 ; \mathrm{H}, 5.87$; $\mathrm{N}, 12.49$.

Butyl 2-(2,4-Dioxo-1-methyl-7-(2-fluorophenyl)-1,4-dihydrofuro[3,2-g]pteridine-3(2H)-yl)acetate (3d). Yield: $3.24 \mathrm{~g}(76 \%)$, light yellow compound, $\mathrm{mp}>300{ }^{\circ} \mathrm{C}$; IR $\left(\mathrm{cm}^{-1}\right): 1752,1720,1676,1504,1456,1361,1276,1199$, 894, 772, 752; ${ }^{1} \mathrm{H}$ NMR $\delta$ (ppm): 8.22-7.84 (m, $1 \mathrm{H}, \mathrm{Ar}$ H-6), 7.63-7.49 (m, 2H, H-6, Ar H-4), 7.46-7.23 (m, 2H, Ar H-3,5), $4.74\left(\mathrm{~s}, 2 \mathrm{H}, \mathrm{NCH}_{2}\right), 4.17(\mathrm{t}, J=6.6 \mathrm{~Hz}, 2 \mathrm{H}$, $\mathrm{OCH}_{2} \mathrm{CH}_{2} \mathrm{CH}_{2} \mathrm{CH}_{3}$ ), 3.69 (s, $3 \mathrm{H}, 1-\mathrm{N}-\mathrm{CH}_{3}$ ), 1.67 (quintet, $J=6.9 \mathrm{~Hz}, 2 \mathrm{H}, \mathrm{OCH}_{2} \mathrm{CH}_{2} \mathrm{CH}_{2} \mathrm{CH}_{3}$ ), 1.42 (sextet, $J=7.3$ $\left.\mathrm{Hz}, 2 \mathrm{H}, \mathrm{OCH}_{2} \mathrm{CH}_{2} \mathrm{CH}_{2} \mathrm{CH}_{3}\right), 0.97\left(\mathrm{t}, J=7.3 \mathrm{~Hz}, 3 \mathrm{H}, \mathrm{OCH}_{2}\right.$ 
$\left.\mathrm{CH}_{2} \mathrm{CH}_{2} \mathrm{CH}_{3}\right) ;{ }^{13} \mathrm{C}$ NMR $\delta$ (ppm): 168.1 (COO, C-8a), 159.7 (d, $J=254.1 \mathrm{~Hz}$, Ar C-2), 159.0 (C-4), 155.3 (d, $J=$ $18.2 \mathrm{~Hz}, \mathrm{C}-7), 150.1$ (C-2), 146.0 (C-9a), 138.3 (C-5a), 133.1 (d, $J=8.9 \mathrm{~Hz}$, Ar C-5), 127.5 (Ar C-6), 125.7 (d, $J=$ $21.2 \mathrm{~Hz}, \mathrm{Ar} \mathrm{C}-1), 125.6$ (C-4a), 117.1 (d, $J=20.7 \mathrm{~Hz}, \mathrm{Ar}$ C-3), $116.4(\mathrm{~d}, J=11.0 \mathrm{~Hz}, \mathrm{Ar} \mathrm{C}-4), 106.4(\mathrm{~d}, J=12.2 \mathrm{~Hz}$, C-6), $65.29\left(\mathrm{OCH}_{2} \mathrm{CH}_{2} \mathrm{CH}_{2} \mathrm{CH}_{3}\right), 43.22\left(\mathrm{NCH}_{2} \mathrm{CO}\right), 30.55$ $\left(\mathrm{N}-\mathrm{CH}_{3}\right), 30.12\left(\mathrm{OCH}_{2} \mathrm{CH}_{2} \mathrm{CH}_{2} \mathrm{CH}_{3}\right), 18.94\left(\mathrm{OCH}_{2} \mathrm{CH}_{2}\right.$ $\left.\mathrm{CH}_{2} \mathrm{CH}_{3}\right), 13.95\left(\mathrm{OCH}_{2} \mathrm{CH}_{2} \mathrm{CH}_{2} \mathrm{CH}_{3}\right) ; \mathrm{LC}-\mathrm{MS} \mathrm{m} / z=426$ $[\mathrm{M}+\mathrm{H}]^{+}$. Anal. Calcd. for $\mathrm{C}_{21} \mathrm{H}_{19} \mathrm{FN}_{4} \mathrm{O}_{5}$ : C, 59.15; H, 4.49; $\mathrm{N}, 13.14$; found: $\mathrm{C}, 59.21 ; \mathrm{H}, 4.53 ; \mathrm{N}, 13.19$.

Butyl 2-(2,4-Dioxo-1-methyl-7-(4-fluorophenyl)-1,4-dihydrofuro $[3,2-g]$ pteridine-3(2H)-yl)acetate (3e). Yield: $3.36 \mathrm{~g}(79 \%)$, light yellow compound, $\mathrm{mp}>300{ }^{\circ} \mathrm{C}$; IR $\left(\mathrm{cm}^{-1}\right): 1719,1679,1603,1502,1359,1279,1194,1157$, 1007, 895, 833, 800, 755; ${ }^{1} \mathrm{H}$ NMR $\delta$ (ppm): 8.12 (d, $J=7.6$ $\mathrm{Hz}, 2 \mathrm{H}, \mathrm{Ar} \mathrm{H}-2,6), 7.79$ (s, $1 \mathrm{H}, \mathrm{H}-6), 7.33(\mathrm{t}, J=7.9 \mathrm{~Hz}$, $2 \mathrm{H}, \mathrm{Ar} \mathrm{H}-3,5), 4.75\left(\mathrm{~s}, 2 \mathrm{H}, \mathrm{NCH}_{2}\right), 4.17(\mathrm{t}, J=6.5 \mathrm{~Hz}, 2 \mathrm{H}$, $\left.\mathrm{OCH}_{2} \mathrm{CH}_{2} \mathrm{CH}_{2} \mathrm{CH}_{3}\right), 3.71$ (s, $\left.3 \mathrm{H}, 1-\mathrm{N}-\mathrm{CH}_{3}\right), 1.66$ (quintet, $\left.J=7.2 \mathrm{~Hz}, 2 \mathrm{H}, \mathrm{OCH}_{2} \mathrm{CH}_{2} \mathrm{CH}_{2} \mathrm{CH}_{3}\right), 1.42\left(\mathrm{~m}, 2 \mathrm{H}, \mathrm{OCH}_{2}\right.$ $\left.\mathrm{CH}_{2} \mathrm{CH}_{2} \mathrm{CH}_{3}\right), 0.97$ (t, $\left.J=7.2 \mathrm{~Hz}, 3 \mathrm{H}, \mathrm{OCH}_{2} \mathrm{CH}_{2} \mathrm{CH}_{2} \mathrm{CH}_{3}\right)$; ${ }^{13} \mathrm{C}$ NMR $\delta$ (ppm): 170.4 (COO), 169.2(C-8a), 166.7 (d, $J$ $=258.7 \mathrm{~Hz}, \operatorname{Ar} \mathrm{C}-4), 160.0$ (C-4), $158.4(\mathrm{C}-7), 150.6$ (C-2), 145.9 (C-9a), 136.6 (C-5a), 129.8 (d, $J=9.6 \mathrm{~Hz}, \mathrm{Ar} \mathrm{C}-2,6$ ), $122.2(\mathrm{~d}, J=2.3 \mathrm{~Hz}, \operatorname{Ar} \mathrm{C}-1), 117.0(\mathrm{C}-4 \mathrm{a}), 116.9$ (d, $J=$ $23.1 \mathrm{~Hz}, \mathrm{Ar} \mathrm{C}-3,5), 96.6(\mathrm{C}-6), 68.2\left(\mathrm{OCH}_{2} \mathrm{CH}_{2} \mathrm{CH}_{2} \mathrm{CH}_{3}\right)$, $43.5\left(\mathrm{NCH}_{2} \mathrm{CO}\right), 30.0\left(\mathrm{~N}-\mathrm{CH}_{3}\right), 29.7\left(\mathrm{OCH}_{2} \mathrm{CH}_{2} \mathrm{CH}_{2} \mathrm{CH}_{3}\right)$, $18.2\left(\mathrm{OCH}_{2} \mathrm{CH}_{2} \mathrm{CH}_{2} \mathrm{CH}_{3}\right), 11.7\left(\mathrm{OCH}_{2} \mathrm{CH}_{2} \mathrm{CH}_{2} \mathrm{CH}_{3}\right)$; LC-MS $m / z=426[\mathrm{M}+\mathrm{H}]^{+}$. Anal. Calcd. for $\mathrm{C}_{21} \mathrm{H}_{19} \mathrm{FN}_{4} \mathrm{O}_{5}$ : C, 59.15; H, 4.49; N, 13.14; found: C, 59.20; H, 4.53; N, 13.19.

Butyl 2-(2,4-Dioxo-7-(2,4-difluorophenyl)-1-methyl -1,4-dihydrofuro[3,2-g]pteridine-3(2H)-yl)acetate (3f). Yield: $3.55 \mathrm{~g}(80 \%)$, light yellow compound, $\mathrm{mp}>300^{\circ} \mathrm{C}$; IR $\left(\mathrm{cm}^{-1}\right)$ : 1750, 1722, 1677, 1601, 1501, 1453, 1362, 1278, 1198, 893, 803, 774, 753; ${ }^{1} \mathrm{H}$ NMR $\delta$ (ppm): 8.32-8.05 (m, 1H, Ar H-6), 7.71 (s, 1H, H-6), 7.58-7.49 (m, 2H, Ar $\mathrm{H}-3,5), 4.76\left(\mathrm{~s}, 2 \mathrm{H}, \mathrm{NCH}_{2}\right), 4.21\left(\mathrm{t}, J=6.6 \mathrm{~Hz}, 2 \mathrm{H}, \mathrm{OCH}_{2}\right.$ $\mathrm{CH}_{2} \mathrm{CH}_{2} \mathrm{CH}_{3}$ ), $3.70\left(\mathrm{~s}, 3 \mathrm{H}, 1-\mathrm{N}-\mathrm{CH}_{3}\right.$ ), 1.71 (quintet, $J=6.9$ $\mathrm{Hz}, 2 \mathrm{H}, \mathrm{OCH}_{2} \mathrm{CH}_{2} \mathrm{CH}_{2} \mathrm{CH}_{3}$ ), 1.44 (sextet, $J=7.3 \mathrm{~Hz}, 2 \mathrm{H}$, $\left.\mathrm{OCH}_{2} \mathrm{CH}_{2} \mathrm{CH}_{2} \mathrm{CH}_{3}\right), 1.00\left(\mathrm{t}, J=7.3 \mathrm{~Hz}, 3 \mathrm{H}, \mathrm{OCH}_{2} \mathrm{CH}_{2}\right.$ $\left.\mathrm{CH}_{2} \mathrm{CH}_{3}\right)$; LC-MS $m / z=444[\mathrm{M}+\mathrm{H}]^{+}$. Anal. Calcd. for $\mathrm{C}_{21} \mathrm{H}_{18} \mathrm{~F}_{2} \mathrm{~N}_{4} \mathrm{O}_{5}$ : $\mathrm{C}, 56.76 ; \mathrm{H}, 4.08 ; \mathrm{N}, 12.61$; found: $\mathrm{C}, 56.81$; $\mathrm{H}, 4.13 ; \mathrm{N}, 12.66$.

Butyl 2-(2,4-Dioxo-1-methyl-7-(4-chlorophenyl)-1,4-dihydrofuro[3,2-g]pteridine-3(2H)-yl)acetate $(3 \mathrm{~g})$. Yield: $3.44 \mathrm{~g}(78 \%)$, light yellow compound, $\mathrm{mp}>300^{\circ} \mathrm{C}$; IR $\left(\mathrm{cm}^{-1}\right): 1751,1719,1679,1509,1361,1275,1198,1019$, 928, 893, 830, 802, 756; ${ }^{1} \mathrm{H}$ NMR $\delta$ (ppm): 8.08 (d, $J=9.2$ $\mathrm{Hz}, 2 \mathrm{H}, \mathrm{Ar} \mathrm{H}-2,6), 7.87$ (s, 1H, H-6), 7.58 (d, $J=9.4 \mathrm{~Hz}$, $2 \mathrm{H}, \mathrm{Ar} \mathrm{H}-3,5), 4.75\left(\mathrm{~s}, 2 \mathrm{H}, \mathrm{NCH}_{2}\right), 4.47-4.03(\mathrm{~m}, 2 \mathrm{H}$, $\left.\mathrm{OCH}_{2} \mathrm{CH}_{2} \mathrm{CH}_{2} \mathrm{CH}_{3}\right), 3.71$ (s, $\left.3 \mathrm{H}, 1-\mathrm{N}-\mathrm{CH}_{3}\right), 1.73-1.55$ (m, $\left.2 \mathrm{H}, \mathrm{OCH}_{2} \mathrm{CH}_{2} \mathrm{CH}_{2} \mathrm{CH}_{3}\right), 1.50-1.36\left(\mathrm{~m}, 2 \mathrm{H}, \mathrm{OCH}_{2}\right.$
$\left.\mathrm{CH}_{2} \mathrm{CH}_{2} \mathrm{CH}_{3}\right), 0.97\left(\mathrm{t}, 3 \mathrm{H}, \mathrm{OCH}_{2} \mathrm{CH}_{2} \mathrm{CH}_{2} \mathrm{CH}_{3}\right) ;{ }^{13} \mathrm{C} \mathrm{NMR}$ $\delta$ (ppm): 170.5 (COO), 167.5 (C-8a), 159.3 (C-4), 159.1 (C-7), 150.7 (C-2), 145.9 (C-9a), 140.4 (Ar C-4), 137.4 (C5a), 129.7 (Ar C-2,6), 127.8 (Ar C-3,5), 124.5 (Ar C-1), 118.3 (C-4a), 97.9 (C-6), $68.2\left(\mathrm{OCH}_{2} \mathrm{CH}_{2} \mathrm{CH}_{2} \mathrm{CH}_{3}\right), 43.5$ $\left(\mathrm{NCH}_{2} \mathrm{CO}\right), 29.9\left(\mathrm{~N}-\mathrm{CH}_{3}\right), 29.7\left(\mathrm{OCH}_{2} \mathrm{CH}_{2} \mathrm{CH}_{2} \mathrm{CH}_{3}\right)$, $18.2\left(\mathrm{OCH}_{2} \mathrm{CH}_{2} \mathrm{CH}_{2} \mathrm{CH}_{3}\right), 11.7 \quad\left(\mathrm{OCH}_{2} \mathrm{CH}_{2} \mathrm{CH}_{2} \mathrm{CH}_{3}\right)$; LC-MS $m / z=442[\mathrm{M}+\mathrm{H}]^{+}$. Anal. Calcd. for $\mathrm{C}_{21} \mathrm{H}_{19} \mathrm{Cl}-$ $\mathrm{N}_{4} \mathrm{O}_{5}:$ C, 56.96; H, 4.32; N, 12.65; found: C, $57.01 ; \mathrm{H}, 4.37$; $\mathrm{N}, 12.69$.

Butyl 2-(7-(4-Bromophenyl)-2,4-dioxo-1-methyl-1,4-dihydrofuro[3,2- $g]$ pteridine-3(2H)-yl)acetate $(3 \mathrm{~h})$. Yield: $3.79 \mathrm{~g}(78 \%)$, light yellow compound, $\mathrm{mp}>300{ }^{\circ} \mathrm{C}$; IR $\left(\mathrm{cm}^{-1}\right): 1748,1718,1678,1505,1453,1361,1275,1203$, $1058,1005,929,894,817,801,756 ;{ }^{1} \mathrm{H}$ NMR $\delta$ (ppm): 8.01 $(\mathrm{d}, J=9.0 \mathrm{~Hz}, 2 \mathrm{H}, \mathrm{Ar} \mathrm{H}-2,6), 7.89$ (s, $1 \mathrm{H}, \mathrm{H}-6), 7.73$ (d, $J=$ $7.9 \mathrm{~Hz}, 2 \mathrm{H}, \mathrm{Ar} \mathrm{H}-3,5), 4.75$ (s, $\left.2 \mathrm{H}, \mathrm{NCH}_{2}\right), 4.21-4.07$ (m, $\left.2 \mathrm{H}, \mathrm{OCH}_{2} \mathrm{CH}_{2} \mathrm{CH}_{2} \mathrm{CH}_{3}\right), 3.71\left(\mathrm{~s}, 3 \mathrm{H}, 1-\mathrm{N}-\mathrm{CH}_{3}\right), 1.75-$ $1.55\left(\mathrm{~m}, 2 \mathrm{H}, \mathrm{OCH}_{2} \mathrm{CH}_{2} \mathrm{CH}_{2} \mathrm{CH}_{3}\right), 1.52-1.36(\mathrm{~m}, 2 \mathrm{H}$, $\left.\mathrm{OCH}_{2} \mathrm{CH}_{2} \mathrm{CH}_{2} \mathrm{CH}_{3}\right), \quad 1.02-0.92 \quad\left(\mathrm{~m}, \quad 3 \mathrm{H}, \quad \mathrm{OCH}_{2} \mathrm{CH}_{2}\right.$ $\left.\mathrm{CH}_{2} \mathrm{CH}_{3}\right) ;{ }^{13} \mathrm{C}$ NMR $\delta$ (ppm): $170.4(\mathrm{COO}), 166.9(\mathrm{C}-8 \mathrm{a})$, 159.5 (C-4), 159.0 (C-7), 150.8 (C-2), 145.9 (C-9a), 137.7 (C-5a), 132.7 (Ar C-2,6), 128.3 (C-1), 127.6 (Ar C-3,5), 125.1 (C-4), 119.4 (C-4a), 98.3 (C-6), $68.2\left(\mathrm{OCH}_{2} \mathrm{CH}_{2}\right.$ $\left.\mathrm{CH}_{2} \mathrm{CH}_{3}\right), 43.6\left(\mathrm{NCH}_{2} \mathrm{CO}\right), 29.9\left(\mathrm{~N}-\mathrm{CH}_{3}\right), 29.7\left(\mathrm{OCH}_{2}\right.$ $\left.\mathrm{CH}_{2} \mathrm{CH}_{2} \mathrm{CH}_{3}\right), 18.2\left(\mathrm{OCH}_{2} \mathrm{CH}_{2} \mathrm{CH}_{2} \mathrm{CH}_{3}\right), 11.8\left(\mathrm{OCH}_{2}\right.$ $\mathrm{CH}_{2} \mathrm{CH}_{2} \mathrm{CH}_{3}$ ); LC-MS $m / z=487[\mathrm{M}+\mathrm{H}]^{+}$. Anal. Calcd. for $\mathrm{C}_{21} \mathrm{H}_{19} \mathrm{BrN}_{4} \mathrm{O}_{5}: \mathrm{C}, 51.76 ; \mathrm{H}, 3.93 ; \mathrm{N}, 11.50$; found: $\mathrm{C}$, 51.82; H, 3.98; N, 11.57.

Butyl 2-(2,4-Dioxo-1-methyl-7-(3-nitropheny)-1,4-dihydrofuro $[3,2-g]$ pteridine-3(2H)-yl)acetate (3i). Yield: $3.53 \mathrm{~g}$ (78\%), light brown compound, mp: $287-289^{\circ} \mathrm{C}$; IR $\left(\mathrm{cm}^{-1}\right): 1713,1674,1505,1349,1315,1282,1211,918,857$, 804, 756, 740, 723, 684; ${ }^{1} \mathrm{H}$ NMR $\delta$ (ppm): 8.89 (s, $1 \mathrm{H}, \mathrm{Ar}$ $\mathrm{H}-2), 8.50$ (d, $J=8.0 \mathrm{~Hz}, 1 \mathrm{H}$, Ar H-6), 8.35 (d, $J=8.4 \mathrm{~Hz}$, $1 \mathrm{H}, \mathrm{Ar} \mathrm{H}-4), 8.19$ (s, 1H, H-6), 7.87 (t, $J=7.1 \mathrm{~Hz}, 1 \mathrm{H}, \mathrm{Ar}$ $\mathrm{H}-5), 4.76\left(\mathrm{~s}, 2 \mathrm{H}, \mathrm{NCH}_{2}\right), 4.18\left(\mathrm{t}, J=6.7 \mathrm{~Hz}, 2 \mathrm{H}, \mathrm{OCH}_{2}\right.$ $\left.\mathrm{CH}_{2} \mathrm{CH}_{2} \mathrm{CH}_{3}\right), 3.72\left(\mathrm{~s}, 3 \mathrm{H}, 1-\mathrm{N}-\mathrm{CH}_{3}\right), 1.81-1.53(\mathrm{~m}, 2 \mathrm{H}$, $\left.\mathrm{OCH}_{2} \mathrm{CH}_{2} \mathrm{CH}_{2} \mathrm{CH}_{3}\right), \quad 1.54-1.34 \quad\left(\mathrm{~m}, \quad 2 \mathrm{H}, \quad \mathrm{OCH}_{2} \mathrm{CH}_{2}\right.$ $\left.\mathrm{CH}_{2} \mathrm{CH}_{3}\right), 0.98\left(\mathrm{t}, J=6.5 \mathrm{~Hz}, 3 \mathrm{H}, \mathrm{OCH}_{2} \mathrm{CH}_{2} \mathrm{CH}_{2} \mathrm{CH}_{3}\right) ;{ }^{13} \mathrm{C}$ NMR $\delta$ (ppm): 170.8 (COO, C-8a), 158.1 (C-4, C-7), 151.2 (C-2), 148.5 (C-9a), 146.3 (Ar C-3), 138.8 (C-5a), 132.2 (Ar C-6), 130.6 (Ar C-5), 129.1 (Ar C-1), 126.1 (Ar C-4), 122.3 (Ar C-2), 120.8 (C-4a), 101.4 (C-6), $68.2\left(\mathrm{OCH}_{2} \mathrm{CH}_{2}\right.$ $\left.\mathrm{CH}_{2} \mathrm{CH}_{3}\right), 43.6\left(\mathrm{NCH}_{2} \mathrm{CO}\right), 29.8\left(\mathrm{OCH}_{2} \mathrm{CH}_{2} \mathrm{CH}_{2} \mathrm{CH}_{3}\right)$, $29.8\left(\mathrm{~N}^{-\mathrm{CH}_{3}}\right), 18.2\left(\mathrm{OCH}_{2} \mathrm{CH}_{2} \mathrm{CH}_{2} \mathrm{CH}_{3}\right), 11.73\left(\mathrm{OCH}_{2}\right.$ $\left.\mathrm{CH}_{2} \mathrm{CH}_{2} \mathrm{CH}_{3}\right) ; \mathrm{LC}-\mathrm{MS} m / z=453[\mathrm{M}+\mathrm{H}]^{+}$. Anal. Calcd. for $\mathrm{C}_{21} \mathrm{H}_{19} \mathrm{~N}_{5} \mathrm{O}_{7}$ : C, 55.63; $\mathrm{H}, 4.22 ; \mathrm{N}, 15.45$; found: $\mathrm{C}$, $55.69 ; \mathrm{H}, 4.28 ; \mathrm{N}, 15.48$.

Butyl 2-(2,4-Dioxo-1-methyl-7-(naphthalen-2-yl)-1,4-dihydrofuro[3,2-g]pteridine-3(2H)-yl)acetate $(3 \mathbf{j})$. Yield: $3.43 \mathrm{~g}(75 \%)$, light orange compound, $\mathrm{mp}>300{ }^{\circ} \mathrm{C}$; IR $\left(\mathrm{cm}^{-1}\right): 1716,1673,1554,1503,1455,1363,1282,1196$, 
945, 906, 800, 748; ${ }^{1} \mathrm{H}$ NMR $\delta$ (ppm): 8.59 (s, $1 \mathrm{H}$, naphthalene $\mathrm{H}-1), 8.11(\mathrm{~d}, J=8.7 \mathrm{~Hz}, 1 \mathrm{H}$, naphthalene $\mathrm{H}-4)$, 8.06$8.00(\mathrm{~m}, 2 \mathrm{H}$, naphthalene $\mathrm{H}-3,8), 7.95-7.83(\mathrm{~m}, 2 \mathrm{H}, \mathrm{H}-6$, naphthalene $\mathrm{H}-5), 7.59(\mathrm{~d}, J=5.2 \mathrm{~Hz}, 2 \mathrm{H}$, naphthalene $\mathrm{H}-6,7), 4.76\left(\mathrm{~s}, 2 \mathrm{H}, \mathrm{NCH}_{2}\right), 4.18\left(\mathrm{t}, J=7.4 \mathrm{~Hz}, 2 \mathrm{H}, \mathrm{OCH}_{2}\right.$ $\left.\mathrm{CH}_{2} \mathrm{CH}_{2} \mathrm{CH}_{3}\right), 3.71\left(\mathrm{~s}, 3 \mathrm{H}, 1-\mathrm{N}-\mathrm{CH}_{3}\right), 1.78-1.56(\mathrm{~m}, 2 \mathrm{H}$, $\left.\mathrm{OCH}_{2} \mathrm{CH}_{2} \mathrm{CH}_{2} \mathrm{CH}_{3}\right), \quad 1.52-1.34 \quad\left(\mathrm{~m}, \quad 2 \mathrm{H}, \quad \mathrm{OCH}_{2} \mathrm{CH}_{2}\right.$ $\left.\underline{\mathrm{CH}}_{2} \mathrm{CH}_{3}\right), 0.98\left(\mathrm{t}, J=7.8 \mathrm{~Hz}, 3 \mathrm{H}, \mathrm{OCH}_{2} \mathrm{CH}_{2} \mathrm{CH}_{2} \mathrm{CH}_{3}\right) ;{ }^{13} \mathrm{C}$ NMR $\delta$ (ppm): 170.2 (COO), 166.6 (C-8a), 158.8 (C-4), 158.3 (C-7), 150.4 (C-2), 145.0 (C-9a), 137.2 (naphthalene C-5a), 134.8 (C-5a), 132.2 (naphthalene C-4a), 129.2 (naphthalene C-4), 128.9 (naphthalene C-8), 128.8 (naphthalene C-5), 127.4 (naphthalene C-6), 127.3 (naphthalene C-3), 127.1 (naphthalene C-7), 123.1 (naphthalene C-2), 121.7 (naphthalene C-1), 118.4 (C-4a), 98.3 (C-6), 68.1 $\left(\mathrm{OCH}_{2} \mathrm{CH}_{2} \mathrm{CH}_{2} \mathrm{CH}_{3}\right), 43.5\left(\mathrm{NCH}_{2} \mathrm{CO}\right), 29.8\left(\mathrm{~N}^{-\mathrm{CH}_{3}}\right)$, $29.7\left(\mathrm{OCH}_{2} \mathrm{CH}_{2} \mathrm{CH}_{2} \mathrm{CH}_{3}\right), \quad 18.3\left(\mathrm{OCH}_{2} \mathrm{CH}_{2} \mathrm{CH}_{2} \mathrm{CH}_{3}\right)$, $11.8\left(\mathrm{OCH}_{2} \mathrm{CH}_{2} \mathrm{CH}_{2} \mathrm{CH}_{3}\right)$; LC-MS $m / z=458[\mathrm{M}+\mathrm{H}]^{+}$. Anal. Calcd. for $\mathrm{C}_{25} \mathrm{H}_{22} \mathrm{~N}_{4} \mathrm{O}_{5}$ : C, 65.49; $\mathrm{H}, 4.84 ; \mathrm{N}, 12.22$; found: $\mathrm{C}, 65.52 ; \mathrm{H}, 4.87 ; \mathrm{N}, 12.25$.

\section{2. X-Ray Diffraction Analysis}

Crystals of compound 2a were monoclinic, $\mathrm{C}_{15} \mathrm{H}_{10} \mathrm{~N}_{4} \mathrm{O}_{3}$, at $20^{\circ} \mathrm{C}, a=7.7443(6) \AA, b=6.4905(4) \AA, c$ $=12.7022(8) \AA, \beta=105.371(7)^{\circ}, V=615.63(7) \AA^{3}, M_{\mathrm{r}}=$ 294.27, $Z=2$, space group P21, $d_{\text {calc. }}=1.587 \mathrm{~g} / \mathrm{cm}^{3}, \mu$
$(\mathrm{MoKa})=0.115 \mathrm{~mm}^{-1}, F(000)=304$. Unit cell parameters and intensities of 5928 reflections (3081 independent, $R_{\text {int }}$ $=0.022$ ) were measured on a Xcalibur-3 diffractometer $(\mathrm{MoK} \alpha)$ radiation, a CCD detector, a graphite monochromator, $\omega$-scanning, $2 \theta_{\max }=60^{\circ}$ ). The structure was deciphered by the direct method using the SHELXTL software package. ${ }^{19}$ The positions of the hydrogen atoms were revealed from the difference synthesis of electron density and refined using the rider model with $U_{\text {iso }}=\mathrm{n} U_{\text {eq }}$ non-hydrogen atom associated with this hydrogen atom $(n=1.5$ for the methyl group and $n=1.2$ for the remaining hydrogen atoms). The hydrogen atom of the amino group was refined in the isotropic approximation. The structure was refined by $\mathrm{F} 2$ by full-matrix least squares in the anisotropic approximation for non-hydrogen atoms up to $w R 2=0.090$ by 3021 reflections $\left(R_{1}=0.035\right.$ by 2646 reflections with $F>$ $4 \sigma(F), S=0.998)$. The atomic coordinates, as well as the complete tables of bond lengths and bond angles, were deposited with the Cambridge Structural Data Bank (e-mail: deposit@ccdc.cam.ac.uk) under the number CCDC 1940140.

\section{Results and Discussion}

The Paal-Knorr synthesis, despite more than a century of experience in use, remains to be one of the most

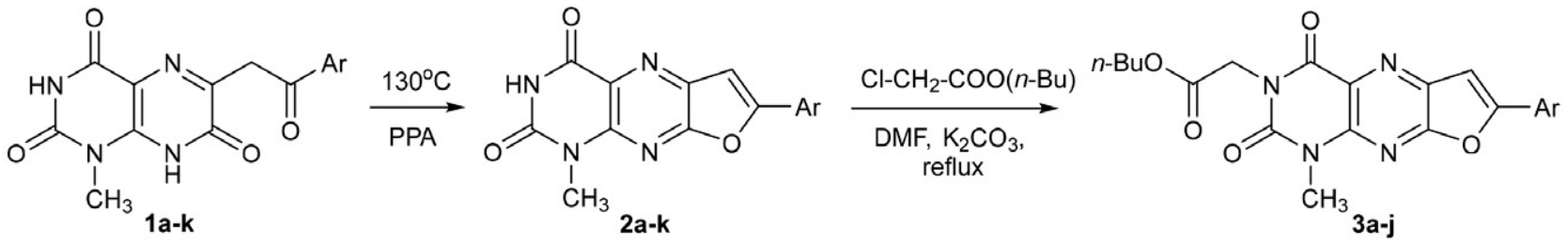

$\mathbf{a}: \mathrm{Ar}=-\mathrm{C}_{6} \mathrm{H}_{5} ; \mathbf{b}: \mathrm{Ar}=-\left(4-\mathrm{CH}_{3}\right) \mathrm{C}_{6} \mathrm{H}_{4} ; \mathbf{c}: \mathrm{Ar}=-\left(4-i-\mathrm{C}_{3} \mathrm{H}_{7}\right) \mathrm{C}_{6} \mathrm{H}_{4} ; \mathbf{d}: \mathrm{Ar}=-(2-\mathrm{F}) \mathrm{C}_{6} \mathrm{H}_{4} ; \mathbf{e}: \mathrm{Ar}=-(4-\mathrm{F}) \mathrm{C}_{6} \mathrm{H}_{4} ; \mathbf{f}: \mathrm{Ar}=-(2,4-\mathrm{F}) \mathrm{C}_{6} \mathrm{H}_{3} ;$

$\mathbf{g}: \mathrm{Ar}=-(4-\mathrm{Cl}) \mathrm{C}_{6} \mathrm{H}_{4} ; \mathbf{h}: \mathrm{Ar}=-(4-\mathrm{Br}) \mathrm{C}_{6} \mathrm{H}_{4} ; \mathbf{i}: \mathrm{Ar}=-\left(3-\mathrm{NO}_{2}\right) \mathrm{C}_{6} \mathrm{H}_{4} ; \mathbf{j}: \mathrm{Ar}=$ naphtalene-2-yl; $\mathbf{k}: \mathrm{Ar}=-\left(4-\mathrm{CH}_{3} \mathrm{O}\right) \mathrm{C}_{6} \mathrm{H}_{4}$.

Scheme 1. The dehydration of 1-methyl-6-(2-oxo-2-arylethyl)pteridine-2,4,7(1H,3H,8H)-triones and the alkylation of 1-methyl-7-arylfuro[3,2-g] pteridine-2,4(1H,3H)-diones<smiles>Cn1c(=O)[nH]c(=O)c2nc(CC(=O)[AlH2])c(=O)[nH]c21</smiles>

A<smiles>C#CC(O)(Br)Cc1nc2c(=O)[nH]c(=O)n(C)c2[nH]c1=O</smiles>

B<smiles></smiles>

C<smiles>Cn1c(=O)[nH]c(=O)c2nc3c(nc21)OC(Br)(CO)C3(C)C</smiles><smiles>Cn1c(=O)[nH]c(=O)c2nc3cc(Br)oc3nc21</smiles>

Scheme 2. The supposed mechanism of 1-methyl-6-(2-oxo-2-arylethyl)pteridine-2,4,7-(1H,3H,8H)-triones dehydration 
effective methods for the formation of five-membered heterocycles with one heteroatom. ${ }^{20}$ Considering the structural similarity of 1-methyl-6-(2-oxo-2-arylethyl)pteridine-2,4,7(1H,3H,8H)-triones $\mathbf{1 a - k}$ with 1,4-dicarbonyl compounds and in the continuation of modification studies of pteridines their dehydration was investigated. It was found that dehydration of compounds $\mathbf{1 a}-\mathbf{k}$ in concentrated sulfuric acid both at room temperature and during heating proceeded doubtfully. In this case, either a mixture of substances difficult to identify was formed, or its tarring occurred. The dehydration reaction was carried out by heating of the starting materials in polyphosphoric acid (Scheme 1). Pure 1-methyl-7-arylfuro[3,2-g]pteridine-2,4 $(1 H, 3 H)$-diones $\mathbf{2 a}-\mathbf{k}$ were formed with high yields.

It should be noted that dehydration of compounds $\mathbf{1 a - k}$ (A in Scheme 2) in the solution of polyphosphoric acid proceeded according to the Paal-Knorr synthesis. ${ }^{21}$ The mechanism of this reaction assumes the nucleophilic attack of the amide fragment oxygen atom of the molecule at the carbon atom of the protonated carbonyl group (B). The oxonium cation $\mathbf{C}$ became aromatic in the result of deprotonation and dehydration with the formation of the final product $\mathbf{E}$.

To increase the solubility of 1-methyl-7-arylfuro[3,2-g]pteridin-2,4(1H,3H)-diones $\mathbf{2 a - k}$ in organic solvents (DMSO, DMF), the next step was to study their alkylation. It was found that alkylation of compounds $2 \mathbf{a}-\mathbf{k}$ by butyl chloroacetate in DMF in the presence of $\mathrm{K}_{2} \mathrm{CO}_{3}$ proceeded by the $\mathrm{N}_{3}$-atom of the heterocycle. Corresponding esters $\mathbf{3 a}-\mathbf{j}$ were formed with satisfactory yields (Scheme 1). In this cases, compounds $\mathbf{3 a}-\mathbf{j}$ have higher solubility in organic solvents.

The formation of compounds $\mathbf{2} \mathbf{a}-\mathbf{k}$ was indicated by ${ }^{1} \mathrm{H}$ NMR spectra. Such singlet signals of proton at the $6^{\text {th }}$ position were recorded in the region of 8.14-7.49 ppm. However, these protons were in some cases (compounds $\mathbf{2 f}$ and $\mathbf{2 h}$ ) recorded as multiplets together with the signals of protons of the substituent at the $7^{\text {th }}$ position. Protons at the $3^{\text {rd }}$ position of compounds $2 \mathbf{a}-\mathbf{k}$ were found in the range of 12.03-11.44 ppm in the low-field part of the ${ }^{1} \mathrm{H}$ NMR spectra. Speaking about compounds $\mathbf{3 a}-\mathbf{j}$, the characteristic signal of the proton at the $6^{\text {th }}$ position was recorded at 8.19-7.69 ppm. At once, in the ${ }^{1} \mathrm{H}$ NMR spectra of com-

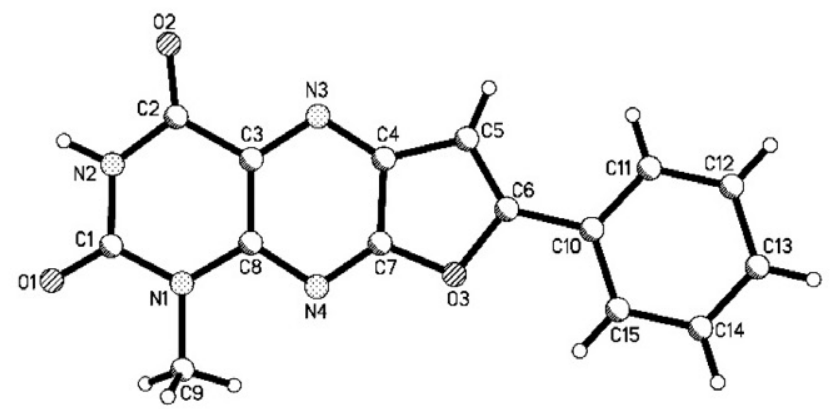

pounds $\mathbf{3} \mathbf{a}-\mathbf{j}$, unlike compounds $\mathbf{2} \mathbf{a}-\mathbf{k}$, there were singlet signals of protons of the $\mathrm{NCH}_{2}$ group at $4.74-4.76 \mathrm{ppm}$ and a series of proton signals of a butoxycarbonyl fragment. Also, in the spectra of compounds $2 \mathbf{a}-\mathbf{k}$ and $3 \mathbf{a}-\mathbf{j}$ there were singlet signals of methyl group protons at 3.57$3.72 \mathrm{ppm}$ and a set of signals corresponding to the substituent at the $7^{\text {th }}$ position. ${ }^{22}$ Additionally, the formation of furo[3,2-g]pteridine-2,4(1H,3H)-diones $\mathbf{2 a - k}$ was confirmed by the ${ }^{13} \mathrm{C}$ NMR spectrometry when studying their more soluble esters 3 . The characteristic signals in the ${ }^{13} \mathrm{C}$ NMR spectra of compounds $\mathbf{3 a}-\mathbf{j}$ were: signals of carbon atom at the $6^{\text {th }}$ position at $106.4-95.1 \mathrm{ppm}$, signals of carbon atoms of the COO- group at 168.1-172.7 ppm and signals of $\mathrm{NCH}_{2} \mathrm{CO}$-fragment at $43.2-43.6 \mathrm{ppm}$. The positions of other signals in the ${ }^{13} \mathrm{C}$ NMR spectra correspond to the proposed structures. ${ }^{23}$

The analysis of the data of the chromato-mass spectra confirmed structure and purity of the compounds $2 \mathbf{a}-\mathbf{k}$ and $3 \mathbf{a}-\mathbf{j}$. An additional analysis of the mass spectra (EI) of compounds 2a and 3a showed the fragmentation of the furo[3,2-g]pteridine system. Thus, the high stability of the molecular ion of compound $2 \mathbf{a}\left([\mathrm{M}]^{\bullet+}, m / z=294, I_{\text {rel }}=\right.$ $66.8 \%$ ), determined its fragmentation along the less aromatic dihydropyrimidine cycle with a step-by-step release of HNCO molecules $\left(\mathrm{F}_{1}, m / z=251, I_{\text {rel }}=12.6 \%\right), \mathrm{CO}\left(\mathrm{F}_{2}\right.$, $\left.m / z=223, I_{\text {rel }}=100 \%\right)$ and the $\mathrm{NCH}_{3} \cdot$ ion $\left(\mathrm{F}_{3}, m / z=194\right.$, $\left.I_{\text {rel }}=10.5 \%\right)$. Formed 6-phenylfuro[2,3-b]pyrazine ion $\left(\mathrm{F}_{3}\right)$ eliminated two $\mathrm{HCN}$ molecules with formation of ions with $\mathrm{F}_{4}\left(m / z=167, I_{\text {rel }}=5.9 \%\right)$ and $\mathrm{F}_{5}\left(m / z=140, I_{\text {rel }}\right.$ $=24.8 \%$ ), while for $\mathrm{F}_{5}$ formation of two alternative fragmentation ions $\left[\mathrm{C}_{6} \mathrm{H}_{5}\right]^{\circ+}\left(\mathrm{m} / z=77, I_{\text {rel }}=25.3 \%\right)$ and $\left[\mathrm{C}_{4} \mathrm{H}_{3} \mathrm{O}\right]^{++}\left(\mathrm{m} / z=67, I_{\text {rel }}=16.8 \%\right)$ was characteristic. Whereas, the molecular ion of ether $\mathbf{3 a}$ was less stable $\left([\mathrm{M}]^{\cdot+}, m / z=408, I_{\text {rel }}=46.6 \%\right)$. The main ways of its fragmentation were associated with the initial elimination of $\mathrm{C}_{4} \mathrm{H}_{9}{ }^{+}\left(\mathrm{F}_{1}, m / z=352, I_{\text {rel }}=10.6 \%\right)$ and $\mathrm{CO}_{2}\left(\mathrm{~F}_{2}, m / z=308\right.$, $\left.I_{\text {rel }}=31.9 \%\right)$. Further degradation of the fragmented ion $\left(\mathrm{F}_{2}\right)$ proceeded similarly to the path described for compound 2a, which led to the appearance of signals with $\mathrm{m} / \mathrm{z}$ $=251\left(I_{\text {rel }}=9.5 \%\right), m / z=223\left(I_{\text {rel }}=23.9 \%\right)$ and $m / z=140$ $\left(I_{\text {rel }}=10.2 \%\right)$.

The final structure of compound 2 a was confirmed by X-ray diffraction study (Fig. 1). It was found that it

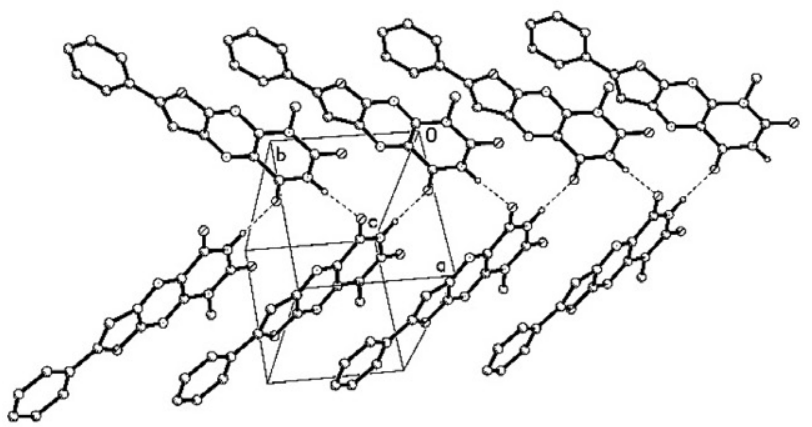

Fig. 1. Molecular structure and packing of molecules in a crystal of compound 2a. 
crystallized in the non-centrosymmetric space group P21, despite the absence of chiral centers in the molecule (Fig. 1).

All non-hydrogen atoms in the molecule lie in the plane with an accuracy of $0.05 \AA$, despite the presence of slight steric repulsion between the atoms of the tricyclic fragment and the phenyl substituent (shortened intramolecular contacts $\mathrm{H}(11) \ldots \mathrm{C}(5) 2.79 \AA$ with the sum of the van der Waals radii ${ }^{24} 2.87 \AA$ and $\mathrm{H}(15) \cdots \mathrm{O}(3) 2.43 \AA$ (2.46 $\AA$ ). In the crystal of molecule $2 \mathbf{a}$ double chains along the crystallographic direction [llll $\left.\begin{array}{lll}0 & 1\end{array}\right]$ were formed due to the intermolecular hydrogen bond $\mathrm{N}(2)-\mathrm{H} \cdots \mathrm{O}(2)^{\prime}(-x,-0.5+$ $y,-z), \mathrm{H} \cdots \mathrm{O} 1.94 \AA$, N-H… $175^{\circ}$ and stacking interactions (the distance between the $\pi$-systems of neighboring molecules was $3.37 \AA$ ).

\section{Conclusion}

Using spectral methods and X-ray diffraction studies, it was found that the dehydration of 1-methyl-6-(2oxo-2-arylethyl)pteridine-2,4,7(1H,3H,8H)-triones proceeded according to the Paal-Knorr synthesis with the formation of the original 1-methyl-7-arylfuro[3,2-g]pteridine-2,4(1H,3H)-diones. For these molecules, the alkylation reaction was studied.

\section{Acknowledgements}

The work was performed with the financial support of «Enamine Ltd» (Kyiv, Ukraine).

\section{References}

1. W. Pfleiderer, Compr. Heterocycl. Chem. II, Bicyclic 6-6 Systems: Pteridines; A. R. Katritzky, C. W. Rees, E. F. V. Scriven, (Eds.): Pergamon Press, 1996, pp. 679-736.

DOI:10.1016/B978-008096518-5.00162-3

2. C. Suckling, C. Gibson, J. Huggan, Compr. Heterocycl. Chem. III, Bicyclic 6-6 Systems: Pteridines; Katritzky, A. R.; Ramsden, C. A.; Taylor, R. J. K. Eds.; Elsevier, 2008, pp. 915-975. DOI:10.1016/B978-008044992-0.00918-4

3. A. V. Gulevskaya, A. F. Pozharskii, Russ. Chem. Rev. 2011, 80(6), 495-529. DOI:10.1070/RC2011v080n06ABEH004168

4. A. A. Sayed, A. H. Elghandour, H. S. Elgendy, Pharma Chem. 2014, 6(3), 194-219

5. C. Suckling, IUBMB Life 2013, 65(4), 283-299.

DOI:10.1002/iub.1148

6. A. Fadda, N. Bayoumy, I. El-Sherbiny, Drug Dev. Ind. Pharm. 2015, 42(7), 1-16. DOI:10.3109/03639045.2015.1108331
7. I. H. El Azab, M. E. Khalifa, A. A. Gobouri, T. A. Altalhi, J. Heterocycl. Chem. 2019, 56, 1352-1361.

DOI:10.1002/jhet.3509

8. M. Mokaber-Esfahani, H. Eshghi, A. Shiri, M. Akbarzadeh, M. Mirzaei, J. Chem. Res. 2015, 39, 216-219.

DOI:10.3184/174751915X14271341601550

9. S. El Kalyoubi, E. Fayed, J. Chem. Res. 2016, 40, 771-777. DOI:10.3184/174751916X14798125870610

10. X. Bi, J. Li, J. Li, W. Shi, Y. Dai, Q. Li, W. Zhang, W. Huang, H. Qian, C. Jiang, Bioorg. Med. Chem. 2019, 27, 2813-2821. DOI:10.1016/j.bmc. 2019.05.006

11. A. Abu-Hashema, M. El-Shazly, Med. Chem. 2018, 14, 356-371. DOI:10.2174/1573406414666180112110947

12. A. A. Ghoneim, N. Ali, A. Elkanzi, R. B. Bakr, J. Taibah. Univ. Sci. 2018, 12(6), 774-782.

DOI:10.1080/16583655.2018.1510163

13. A. Kiryanov, S. Natala, B. Jones, C. McBride, V. Feher, B. Lam, Y. Liu, K. Honda, N. Uchiyama, T. Kawamoto, Y. Hikichi, L. Zhang, D. Hosfield, R. Skene, H. Zou, J. Stafford, X. Cao, T. Ichikawa, Bioorg. Med. Chem. Lett. 2017, 27, 1311-1315. DOI:10.1016/j.bmcl.2016.10.009

14. K. Ishimoto, K. Nakaoka, O. Yabe, A. Nishiguchi, T. Ikemoto, Tetrahedron 2018, 74, 5779-5790.

DOI:10.1016/j.tet.2018.08.020

15. A. A. Wiles, B. Fitzpatrick, N. A. McDonald, M. M. Westwater, De-L. B. Long, Ebenhoch, V. M. I. D. Rotello, W. Samuel, G. Cooke, RSC Adv. 2016, 6, 7999-8005.

DOI:10.1039/C5RA22402K

16. I. H. El Azab, N. A. A. Elkanzi, A. A. Gobouri, J. Heterocycl. Chem. 2018, 55, 65-76. DOI:10.1002/jhet.2978

17. V. A. Mamedov, N. A. Zhukova, A. T. Gubaidullin, V. V. Syakaev, M. S. Kadyrova, T. N. Beschastnova, O. B. Bazanova, I. Kh. Rizvanov, S. K. Latypov, J. Org. Chem. 2018, 83, 14942-14964. DOI:10.1021/acs.joc.8b02161

18. M. S. Kazunin, O. Yu. Voskoboynik, I. S. Nosulenko, G. G. Berest, T. Sergeieva, S. Okovytyy, O. V. Karpenko, B. O. Priimenko, S. I. Kovalenko, J. Heterocycl. Chem. 2018, 4, 1033-1041. DOI:10.1002/jhet.3135

19. G. M. Sheldrick Acta Cryst. 2008, A64, 112-122. DOI:10.1107/S0108767307043930

20. A. R. Katritzky, C. W. Rees, In Comprehensive Heterocyclic Chemistry; (Eds.): Pergamon Press, Oxford, 1984, 4, p. 705.

21. A. Venkateraman, A. Kalyani, J. Org. Chem. 1995, 60, 301-307. DOI:10.1021/jo00107a006

22. O. I. El-Sabbagh, M. E. El-Sadek, S. El-Kalyoubi, I. Ismail, Arch. Pharm. Chem. Life Sci. 2007, 340, 26-31.

DOI:10.1002/ardp.200600149

23. N. E. Jacobsen, NMR spectroscopy explained: simplified theory, applications and examples for organic chemistry and structural biology; John Wiley \& Sons, Inc., 2007, p. 688.

24. Yu. V. Zefirov, Crystallography 1997, 42(5), 936-958. 


\section{Povzetek}

$\mathrm{V}$ članku predstavljamo enostavno in učinkovito metodo sinteze doslej neopisanih 1-metil-7-arilfuro[3,2-g]pteridin-2,4(1H,3H)-dionov s pomočjo dehidratacije ustreznih 1-metil-6-fenacilpteridin-2,4,7(1H,3H,8H)-trionov. Pokazali smo, da njihovo alkiliranje $\mathrm{z}$ butil kloroacetatom $\mathrm{v}$ bazičnem poteka na $\mathrm{N}_{3}$-atomu heterocikla. Strukturo in čistočo pripravljenih produktov smo dokazali z IR, ${ }^{1} \mathrm{H}$ in ${ }^{13} \mathrm{C}$ NMR spektroskopijo, plinsko kromatografijo-masno spektrometrijo, masno spektrometrijo in tudi z rentgensko difrakcijsko analizo. Opisujemo tudi predlagani mehanizem dehidratacije.

Except when otherwise noted, articles in this journal are published under the terms and conditions of the Creative Commons Attribution 4.0 International License 